\title{
Pacific
}

Journal of

Mathematics

\section{INEQUALITIES FOR QUASICONFORMAL MAPPINGS IN SPACE}

Glen Douglas Anderson,

MAVINA KRISHNA VAMANAMURTHY AND MATTI VUORINEN 


\section{INEQUALITIES FOR QUASICONFORMAL MAPPINGS IN SPACE}

\section{G. D. Anderson, M. K. Vamanamurthy and M. Vuorinen}

A new lower bound for the conformal capacity of the Grötzsch ring and sharp bounds for the radial distortion of a quasiconformal automorphism of the unit ball are obtained in $n$-space, $n \geq 2$.

1. Introduction. The conformal capacities of the Grötzsch and Teichmüller extremal rings in $\mathbb{R}^{n}, n \geq 2$ (see $\S 2$ ), are denoted by

$$
\gamma_{n}(s)=\operatorname{cap} R_{G, n}(s) \text { and } \tau_{n}(t)=\operatorname{cap} R_{T, n}(t),
$$

respectively, where $s>1$ and $t>0$. The modulus $M_{n}(r)$ of the Grötzsch ring $R_{G, n}(1 / r), 0<r<1$, is defined by

$$
\gamma_{n}(1 / r)=\omega_{n-1} M_{n}(r)^{1-n},
$$

where $\omega_{n-1}$ is the $(n-1)$-dimensional measure of the unit sphere $S^{n-1}$ in $\mathbb{R}^{n}$. The capacities in (1.1) are related [G, $\left.\S 18\right]$ by

$$
\gamma_{n}(s)=2^{n-1} \tau_{n}\left(s^{2}-1\right), \quad s>1 .
$$

For $K>0$ define increasing homeomorphisms $\varphi_{K, n}$ and $\psi_{K, n}$ from $(0,1)$ onto $(0,1)$ by

$$
\left\{\begin{array}{l}
\varphi_{K, n}(r)=1 / \gamma_{n}^{-1}\left(K \gamma_{n}(1 / r)\right)=M_{n}^{-1}\left(\alpha M_{n}(r)\right), \\
\psi_{K, n}(r)=\left(1-\varphi_{1 / K, n}^{2}\left(r^{\prime}\right)\right)^{1 / 2}
\end{array}\right.
$$

where $r^{\prime}=\sqrt{1-r^{2}}$ and $\alpha=K^{1 /(1-n)}$. Given a domain $D$ in $\mathbb{R}^{n}$, for $K \geq 1$ let $Q C_{K}(D)$ and $Q R_{K}(D)$ denote the class of all $K$ quasiconformal and $K$-quasiregular mappings, respectively, of $D$ into itself [V1], [Vu2]. For $K \geq 1,0<r<1$, define [AVV2]

$$
\begin{aligned}
\varphi_{K, n}^{*}(r)=\sup \{|f(x)|:|x|=r, & \left.f(0)=0, f \in Q C_{K}\left(B^{n}\right)\right\}, \\
\varphi_{1 / K, n}^{*}(r)=\inf \{|f(x)|:|x|=r, & f(0)=0, \\
& \left.f \in Q C_{K}\left(B^{n}\right), f\left(B^{n}\right)=B^{n}\right\} .
\end{aligned}
$$

We extend the functions in (1.4), (1.5), (1.6) to [0,1] by defining them to be 0 at 0 and 1 at 1 . For $n \geq 2, K \geq 1,0<r<1$, these 
distortion functions are related by the inequalities [Vu1, 3.5, 5.20], [AVV2, (1.4), Theorem 2.24]

$$
\left\{\begin{array}{l}
\varphi_{K, n}^{*}(r) \leq \min \left\{\varphi_{K, n}(r), \psi_{K, n}(r)\right\}, \\
\varphi_{1 / K, n}^{*}(r) \geq \max \left\{\varphi_{1 / K, n}(r), \psi_{1 / K, n}(r)\right\} .
\end{array}\right.
$$

Each of these three functions is increasing from $[0,1]$ onto $[0,1]$ (see $\S 3$ below). For $n=2$ the inequalities in (1.7) reduce to equalities.

It is well known that $M_{n}(r)+\log r$ is monotone decreasing on $(0,1)$ [T, p. 632], [G]. The so-called Grötzsch ring constant $\lambda_{n}$ defined by

$$
\log \lambda_{n}=\lim _{r \rightarrow 0+}\left(M_{n}(r)+\log r\right)
$$

satisfies $\lambda_{2}=4[\mathbf{T}],\left[\mathbf{L V},(2.11)\right.$, p. 62] and $\lambda_{n} \in\left(2 e^{0.76(n-1)}, 2 e^{n-1}\right)$ for $n \geq 3$ [G], [AVV4, pp. 120-121], [AF].

The main purpose of this paper is to show how one can translate information about the special function $M_{n}(r)$ into information about geometric properties of quasiconformal mappings. An important tool is the following result, which improves the above-mentioned monotone property of $M_{n}(r)+\log r$.

1.9. TheOREM. For each $n \geq 2$, the function

$$
f_{n}(r) \equiv M_{n}(r)+\log \left(\frac{r}{1+r^{\prime}}\right)
$$

is strictly decreasing from $(0,1)$ onto $\left(0, \log \left(\lambda_{n} / 2\right)\right)$, where $\lambda_{n}$ is as in (1.8) and $r^{\prime}=\left(1-r^{2}\right)^{1 / 2}$. Moreover, $f_{2}(r)$ is strictly concave on $(0,1)$.

The next result is an immediate consequence of Theorem 1.9 (cf. [G, Lemma 8], [AVV4, Corollary 2.30]).

1.10. Corollary. For each $n \geq 2,0<r<1, r^{\prime}=\sqrt{1-r^{2}}$,

$$
\begin{gathered}
M_{n}(r)<\log \left(\frac{\lambda_{n}}{2} \frac{1+r^{\prime}}{r}\right)<\log \frac{\lambda_{n}}{r}, \\
\frac{M_{n}(r)}{r \log r} \leq \frac{d}{d r} M_{n}(r) \leq-\frac{1}{r r^{\prime}} .
\end{gathered}
$$

The usual method of obtaining lower bounds for the capacity of a ring is to use spherical symmetrization [G] together with the extremal property of the Teichmüller ring and the fundamental inequality $M_{n}(r) \leq \log \left(\lambda_{n} / r\right)$. By virtue of Corollary $1.10(1)$, all such earlier bounds can now be improved. A graphical comparison of some bounds for $\gamma_{3}(1 / r)$ appears in Figure 1 in $\S 2$. 
From Theorem 1.9 and Corollary $1.10(1)$ we shall derive several inequalities for the distortion functions in (1.4), (1.5), and (1.6). These functions are interesting not only for studying the radial behavior of mappings in $Q C_{K}\left(B^{n}\right)$, but also as means of expressing other special functions.

1.11. Theorem. For $n \geq 2$ and $K \geq 1$ let $\alpha=K^{1 /(1-n)}=1 / \beta$, $L=\left(\lambda_{n} / 2\right)^{1-\alpha}, l=\left(\lambda_{n} / 2\right)^{1-\beta}, l_{1}=2 l /\left(1+l^{2}\right)$. Then

$$
\varphi_{K, n}(r) \leq \tanh \left(2 \operatorname{arctanh}\left(L A(r)^{\alpha}\right)\right)
$$

for $r \in\left(0, l_{1}\right)$, and

$$
\varphi_{1 / K, n}(r) \geq \tanh \left(2 \operatorname{arctanh}\left(l A(r)^{\beta}\right)\right)
$$

for all $r \in(0,1)$, where $A(r)=r /\left(1+r^{\prime}\right), r^{\prime}=\sqrt{1-r^{2}}$. For $K=1$, both (1) and (2) reduce to equality.

The following local Hölder continuity theorem simplifies and improves earlier results in [G], [R, pp. 82-83], [MRV], [AVV2], [Ca].

1.12. Theorem. For $K \geq 1, n \geq 2$ let $f \in Q R_{K}\left(B^{n}\right), 0<r<1$, $\alpha=K^{1 /(1-n)}$, and let $\lambda_{n}$ be as in (1.8).

(1) If $x, y \in \bar{B}^{n}(r)$ then

$$
|f(x)-f(y)| \leq \lambda_{n}^{1-\alpha}\left(1-r^{2}\right)^{-\alpha}|x-y|^{\alpha} .
$$

(2) If $x \in \bar{B}^{n}(r)$ and $y \in B^{n}$, then

$$
|f(x)-f(y)| \leq 2^{\alpha} \lambda_{n}^{1-\alpha}(1-r)^{-\alpha}|x-y|^{\alpha} .
$$

Next, the hyperbolic metric $\rho(x, y)$ on $B^{n}$ is given by

$$
\tanh ^{2} \frac{\rho(x, y)}{2}=\frac{|x-y|^{2}}{|x-y|^{2}+\left(1-|x|^{2}\right)\left(1-|y|^{2}\right)}
$$

for $x, y \in B^{n}$ (cf. [B, p. 40], [Vu2, 2.47]). The following distortion theorem for the hyperbolic metric is a consequence of Theorem 1.9.

1.14. Theorem. For $K \geq 1, n \geq 2$, let $f \in Q R_{K}\left(B^{n}\right)$. Then for all $x, y \in B^{n}$,

$$
\tanh \frac{\rho^{\prime}}{4} \leq\left(\frac{\lambda_{n}}{2}\right)^{1-\alpha}\left(\tanh \frac{\rho}{4}\right)^{\alpha} \leq K\left(\tanh \frac{\rho}{4}\right)^{\alpha} \leq K\left(\tanh \frac{\rho}{4}\right)^{1 / K}
$$


where $\rho=\rho(x, y), \rho^{\prime}=\rho(f(x), f(y))$, and $\alpha=K^{1 /(1-n)}$. These estimates are sharp as $K$ tends to 1 .

1.15. Corollary. For $K \geq 1, n \geq 2$, let $f \in Q C_{K}\left(B^{n}\right)$. Then for all $x, y \in B^{n}$,

$$
\tanh \frac{\rho^{\prime}}{4} \leq \min \{2, K\}\left(\tanh \frac{\rho}{4}\right)^{1 / K},
$$

where $\rho=\rho(x, y)$ and $\rho^{\prime}=\rho(f(x), f(y))$.

The results in $\S 3$ will show that for $n \geq 3$ the functions $\varphi_{K, n}(r)$, $\varphi_{K, n}^{*}(r)$, and $\psi_{K, n}(r)$ in (1.7) behave differently and that the behavior of $\varphi_{K, n}^{*}(r)$ differs drastically from that of $\varphi_{K, 2}(r)$ (cf. 3.7(1) and 3.14).

In $\S 4$ we study the linear dilatation

$$
\begin{aligned}
& H_{n}(K)=\sup \left\{\frac{|f(x)|}{|f(y)|}:|x|=|y|>0,\right. \\
& \left.\qquad f \in Q C_{K}\left(\mathbb{R}^{n}\right), f(0)=0\right\},
\end{aligned}
$$

obtaining the following asymptotic estimate.

1.17. TheOrem. For each $K>1, \lim \sup _{n \rightarrow \infty} H_{n}(K) \leq K^{4}$.

An explicit expression for $H_{n}(K)$ is known only for $n=2$, and

$$
H_{2}(K)=\left(\frac{\mu^{-1}(\pi /(2 K))}{\mu^{-1}(\pi K / 2)}\right)^{2}
$$

$[\mathbf{L V},(6.4)$, p. 81] is also denoted by $\lambda(K)$. It follows from [AVV5, Theorems 1.1, 1.2] that

$$
K^{\pi \sqrt{K}}<e^{\pi(K-1)}<\lambda(K)=H_{2}(K)<e^{\pi(K-(1 / K))}
$$

for $K>1$ and that $(\lambda(K))^{1 / K}$ is strictly increasing to $e^{\pi}$ as $K$ tends to $\infty$. Thus 1.18 and Theorem 1.17 imply that

$$
H_{n}(K)<\left(1+\frac{\log H_{2}(K)}{\pi}\right)^{4}<\left(H_{2}(K)\right)^{4 / \pi}, \quad K>1,
$$

for $n$ sufficiently large. On the other hand by [AVV1, Theorem 1.14], $H_{n}(K) \geq \lambda\left(K^{1 /(n-1)}\right)>1$ for each $K>1$ and $n \geq 2$. Whether the upper bound in Theorem 1.17 can be replaced by 1 remains an open problem (see 4.12(1) below). In any case, Theorem 1.17 shows that quasiconformal mappings become more rigid in high dimensions. If 
one wants to extend the theory of quasiconformal mappings to infinitedimensional Banach spaces then the definition of a $K$-quasiconformal mapping must be chosen carefully, as e.g. in J. Väisälä [V3].

1.20. Notation. For $n \geq 2, \mathbb{R}^{n}$ denotes the $n$-dimensional euclidean space, and $\overline{\mathbb{R}}^{n}$ its one-point compactification $\mathbb{R}^{n} \cup\{\infty\}$. Unit vectors along the coordinate axes in $\mathbb{R}^{n}$ are denoted by $e_{1}, e_{2}, \ldots, e_{n}$. For $x$ in $\mathbb{R}^{n}$ and $r>0$ we let $B^{n}(x, r)=\left\{z \in \mathbb{R}^{n}:|x-z|<\right.$ $r\}, S^{n-1}(x, r)$ its boundary sphere, $B^{n}(r)=B^{n}(0, r), S^{n-1}(r)=$ $S^{n-1}(0, r), B^{n}=B^{n}(1)$, and $S^{n-1}=S^{n-1}(1)$. For any $E \subset \overline{\mathbb{R}}^{n}$ we let $\bar{E}$ denote its closure. For $a, b \in \mathbb{R}^{n}$ we let $[a, b]=$ $\{(1-t) a+t b: 0 \leq t \leq 1\}$ and for $a \in \mathbb{R}^{n} \backslash\{0\},[a, \infty)=$ $\{$ ta: $t \geq 1\},[a, \infty]=[a, \infty) \cup\{\infty\}$. Whenever $0<r<1$, by $r^{\prime}$ we shall mean $\sqrt{1-r^{2}}$.

Given $E, F$, and $G$, subsets of $\overline{\mathbb{R}}^{n}$, we let $\Delta(E, F ; G)$ denote the family of all curves joining $E$ and $F$ in $G$ [V1]. The conformal modulus of a family of curves $\Gamma$ in $\overline{\mathbb{R}}^{n}$ is defined by

$$
M(\Gamma)=\inf _{\rho} \int_{\mathbb{R}^{n}} \rho^{n} d m,
$$

where $d m$ represents $n$-dimensional Lebesgue measure and the infimum is taken over all nonnegative Borel-measurable functions $\rho$ satisfying $\int_{\gamma} \rho d s \geq 1$ for each locally rectifiable curve $\gamma \in \Gamma$ [V1].

Let $G$ be a domain (open connected set) in $\mathbb{R}^{n}$. A continuous function $f: G \rightarrow \mathbb{R}^{n}$ is said to be $K$-quasiregular, $1 \leq K<\infty$, if $f$ is $A C L^{n}$, i.e. $f$ is absolutely continuous on almost all lines parallel to the coordinate axes and the first partial derivatives are locally $L^{n}$ integrable, and if

$$
\left\{\begin{array}{l}
\left|f^{\prime}(x)\right|^{n} \leq K J_{f}(x), \\
J_{f}(x) \leq K l\left(f^{\prime}(x)\right)^{n},
\end{array}\right.
$$

$$
\begin{aligned}
& \left|f^{\prime}(x)\right| \equiv \max _{|h|=1}\left|f^{\prime}(x) h\right|, \\
& \quad l\left(f^{\prime}(x)\right)^{n} \equiv \min _{|h|=1}\left|f^{\prime}(x) h\right|
\end{aligned}
$$

hold a.e. in $G$. Here $f^{\prime}(x)$ denotes the formal derivative of $f$ at $x$, i.e. $f^{\prime}(x) e_{i}=\nabla f_{i}, i=1, \ldots, n, f=\left(f_{1}, f_{2}, \ldots, f_{n}\right)$, and $J_{f}(x)=\operatorname{det}\left(f^{\prime}(x)\right)$. A $K$-quasiregular homeomorphism is called $K$ quasiconformal. A mapping $f$ is called quasiregular or quasiconformal if it is $K$-quasiregular or $K$-quasiconformal, respectively, for some $K \in[1, \infty)$. For properties of these mappings the reader is referred to [V1], [V2], [Vu2].

Acknowledgments. The research of the first two authors was supported in part by grants from the University of Auckland and the New Zealand Mathematical Society, and that of the third author by 
the Alexander von Humboldt Foundation and the Academy of Finland. The first author completed his research during his visit at Indiana University in the ITM/MUCIA Cooperative Program in Malaysia. The third author wishes to thank Professors Ch. Pommerenke and J. Becker for their kind help and hospitality during his visit in 1988-89 at the Technical University of Berlin as a Humboldt Fellow.

2. Modulus of the Grötzsch ring. A ring $R=R\left(C_{0}, C_{1}\right)$ in $\overline{\mathbb{R}}^{n}$ is a domain whose complement consists of two components $C_{0}$ and $C_{1}$, where $\infty \notin C_{0}$. Two extremal rings, the Grötzsch ring

$$
R_{G, n}(s)=\overline{\mathbb{R}}^{n} \backslash\left(\bar{B}^{n} \cup\left[s e_{1}, \infty\right]\right), \quad s>1,
$$

and the Teichmüller ring

$$
R_{T, n}(t)=\overline{\mathbb{R}}^{n} \backslash\left(\left[-e_{1}, 0\right] \cup\left[t e_{1}, \infty\right]\right), \quad t>0,
$$

are important in the study of distortion [G], [Vu1], [Vu2], [AVV1], [AVV2], [AVV5].

The conformal capacity cap $R$ and modulus mod $R$ of a ring $R=$ $R\left(C_{0}, C_{1}\right)$ are given [V1] by

$$
\operatorname{cap} R=M(\Gamma)=\omega_{n-1}(\bmod R)^{1-n},
$$

where $\Gamma=\Delta\left(C_{0}, C_{1} ; R\right)$ and $\omega_{n-1}$ is the $(n-1)$-dimensional measure of $S^{n-1}$.

The function $M_{2}(r)$ defined in formula (1.2), usually denoted by $\mu(r)$, is given explicitly [LV, $(2.2)$, p. 60] by

$$
\mu(r)=\frac{\pi}{2} \frac{\mathscr{K}^{\prime}(r)}{\mathscr{K}(r)},
$$

where

$\mathscr{K}(r)=\int_{0}^{\pi / 2}\left(1-r^{2} \sin ^{2} t\right)^{-1 / 2} d t, \quad \mathscr{K}^{\prime}(r)=\mathscr{K}\left(r^{\prime}\right), \quad r^{\prime}=\sqrt{1-r^{2}}$,

are complete elliptic integrals. We also use the dual integrals

$$
\mathscr{E}(r)=\int_{0}^{\pi / 2}\left(1-r^{2} \sin ^{2} t\right)^{1 / 2} d t, \quad \mathscr{E}^{\prime}(r)=\mathscr{E}\left(r^{\prime}\right) .
$$

The next result is an analog of l'Hôpital's rule and will be useful in establishing monotoneity of a ratio of two functions.

2.2. Lemma. For $-\infty<a<b<\infty$ let $f, g:[a, b) \rightarrow \mathbb{R}$ be differentiable functions such that $g^{\prime}(x) \neq 0$ for $x \in(a, b)$. 
If $f^{\prime}(x) / g^{\prime}(x)$ is increasing (decreasing) on $(a, b)$ then so is $(f(x)-f(a)) /(g(x)-g(a))$.

Proof. We may assume that $g^{\prime}(x)>0$ for all $x \in(a, b)$ and that $f^{\prime}(x) / g^{\prime}(x)$ is increasing on $(a, b)$. By the Cauchy mean value theorem, for $x \in(a, b)$ there exists $y \in(a, x)$ such that

$$
\frac{f(x)-f(a)}{g(x)-g(a)}=\frac{f^{\prime}(y)}{g^{\prime}(y)} \leq \frac{f^{\prime}(x)}{g^{\prime}(x)},
$$

which implies that $(f(x)-f(a)) /(g(x)-g(a))$ has a positive derivative on $(a, b)$.

2.3. Proof of Theorem 1.9. The monotoneity appears in [A1, Theorem 5, p. 15], but for completeness we include the proof here. For $0<a<b<1$ let $R$ be the ring whose boundary components are $S^{n-1}$ and the segment $\left[-a e_{1}, a e_{1}\right]$. Then $S^{n-1}(a / b)$ separates $R$ into two rings $R_{1}$ and $R_{2}$, where $R_{1}$ is conformally equivalent to $R_{G, n}\left(\left(1+b^{2}\right) / 2 b\right)$ and $R_{2}$ is the spherical annulus $\{x: a / b<|x|<$ 1 \}. By superadditivity of the modulus [F] we get $\bmod R \geq \bmod R_{1}$ $+\bmod R_{2}$, that is,

$$
M_{n}\left(\frac{2 a}{1+a^{2}}\right) \geq M_{n}\left(\frac{2 b}{1+b^{2}}\right)+\log \frac{b}{a} .
$$

Next, putting $r_{1}=2 a /\left(1+a^{2}\right), r_{2}=2 b /\left(1+b^{2}\right)$, we conclude that $f_{n}$ is decreasing. The limit as $r$ tends to 0 follows from (1.8), while the limit at 1 is trivial. In [A2, $\S 8]$ a different proof of this monotoneity is based on Hölder's inequality, which, by [A2, Lemma 4], cannot reduce to equality; hence the monotoneity is strict.

Next, let $n=2$ and $f(r)=f_{2}(r)$. Then by [AVV6, Lemma 2.1(2)],

$$
f^{\prime}(r)=\frac{1}{r r^{\prime}}\left(1-\frac{\pi^{2}}{4 r^{\prime} \mathscr{K}(r)^{2}}\right),
$$

which is negative for $0<r<1$ since $r^{\prime} \mathscr{K}(r)^{2}$ is strictly decreasing on $(0,1)$ [AVV6, Theorem 2.2(3)] and $\mathscr{K}(0)=\pi / 2$. Thus $f$ is strictly decreasing on $(0,1)$. Next, $-f^{\prime}(r)=g(r) h(r) / r$, where

$$
g(r)=\frac{\frac{\pi}{2 \sqrt{r^{\prime} \mathscr{K}(r)}}+1}{r^{\prime}} \text { and } h(r)=\frac{\pi}{2 \sqrt{r^{\prime} \mathscr{K}(r)}}-1 .
$$

Since $g(r)$ is increasing [AVV6, Theorem 2.1(3)], we need only prove that $h(r) / r$ is increasing, and by Lemma 2.2 it is sufficient to show 
that $h^{\prime}(r)$ is increasing. First by [BF, 710.00]

$\frac{h^{\prime}(r)}{1+h(r)}=\frac{1}{2 r^{\prime 2} \mathscr{K}} \cdot \frac{H(r)}{r}, \quad$ where $H(r)=(\mathscr{K}-\mathscr{E})-\left(\mathscr{E}-r^{\prime 2} \mathscr{K}(r)\right)$.

By [AVV6, Theorem 2.2(3)] the first factor is increasing; thus by Lemma 2.2 it is sufficient to show that $H^{\prime}(r)$ is increasing. Now, by [BF, 710.04-.05], $H^{\prime}(r)=\left(\mathscr{E}-r^{\prime 2} \mathscr{K}\right) r r^{\prime-2}$, which is clearly increasing.

2.4. Remark. For $0<r<1$, by [A1, Theorem 2], [G, Lemma 8] (cf. [AVV4, (1.10), (1.11)]), and 1.10(1) we have

$$
\left\{\begin{array}{l}
2^{n-1} c_{n} \log \frac{1+r}{1-r} \leq \gamma_{n}(1 / r) \leq 2^{n-1} c_{n} \mu\left(\frac{1-r}{1+r}\right), \\
\omega_{n-1}\left(\log \frac{\lambda_{n}\left(1+r^{\prime}\right)}{2 r}\right)^{1-n} \leq \gamma_{n}(1 / r) \leq \omega_{n-1} \mu(r)^{1-n},
\end{array}\right.
$$

where $c_{n}$ is a constant [V1, p. 31]. Here equality holds on the right sides for $n=2$. Let $f(r), g(r)$ denote the minorants and $F(r)$, $G(r)$ the majorants in $(2.5)$ and let $h(r)=\omega_{n-1}\left(\log \left(\lambda_{n} / r\right)\right)^{1-n}$. Then, for $n=3$, the graphs of these functions are in Figure 1, where the graph of $\gamma_{3}(1 / r)$ lies in the shaded region.

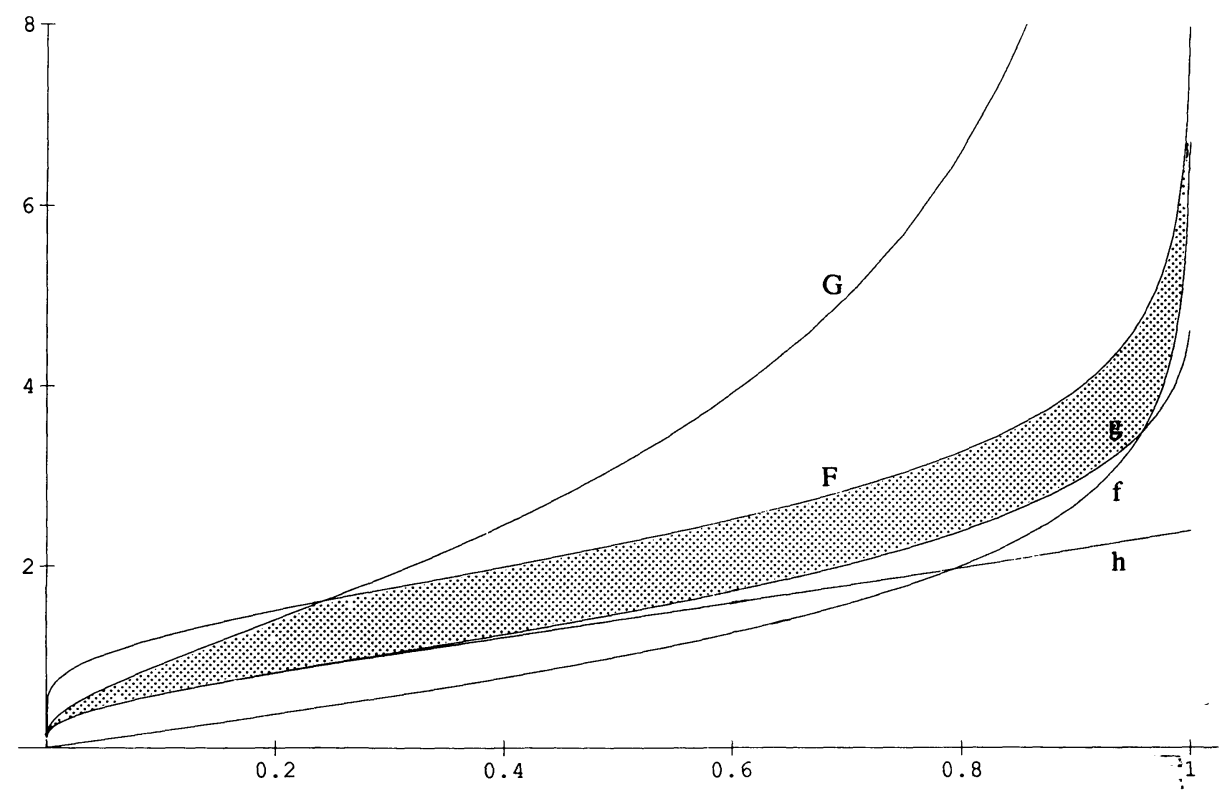

FIGURE 1. Bounds for $\gamma_{3}(1 / r), 0<r<1$

Lower: $f(r)=4 c_{3} \log \frac{1+r}{1-r}, g(r)=\frac{4 \pi}{\log ^{2}\left(4.9501\left(1+r^{\prime}\right) / r\right)}, h(r)=\frac{4 \pi}{\log ^{2}(9.9002 / r)}$, Upper: $F(r)=4 c_{3} \mu\left(\frac{1-r}{1+r}\right), G(r)=\frac{4 \pi}{\mu(r)^{2}}$. 
From Figure 1 we see that the new lower bound $g(r)$ for $\gamma_{3}(1 / r)$ given by Corollary $1.10(1)$ is the best of the present lower bounds when $0<r<r_{0}, r_{0} \approx 0.95$.

\subsection{THEOREM. For $n \geq 2$ the function}

$$
f_{n}(r) \equiv M_{n}(r) \log ((1+\sqrt{r}) /(1-\sqrt{r}))
$$

is strictly increasing from $(0,1)$ onto $I_{n}$, where $I_{2}=\left(0, \pi^{2} / 2\right)$ and $I_{n}=(0, \infty)$ for $n \geq 3$.

Proof. First, by [LV, (2.3), p. 60], $f_{2}(r)=\mu(t) \log ((1+t) /(1-t))$, where $t=2 \sqrt{r} /(1+r)$, and the result follows from [AVV4, Lemma 2.6(4)]. Hence for $n \geq 3$ the result follows from [AVV4, Lemma $2.6(5)]$.

As shown in [AVV4, 1.21], the function $M_{n}$ satisfies several nonlinear functional inequalities. Next we shall prove additional results of this type.

2.7. TheOREM. For each $n \geq 2$ and $s, t \in(0,1)$,

$$
M_{n}\left(\frac{2 s}{1+s^{2}}\right)+M_{n}\left(\frac{2 t}{1+t^{2}}\right) \leq 2 M_{n}\left(\frac{2 \sqrt{s t}}{1+s t}\right) .
$$

There is equality when $s=t$.

Proof. The statement about equality is trivial. For the inequality, let $R$ denote the ring in $\mathbb{R}^{n}$ whose complementary components are the sets $\left[-e_{1} /(s t), \infty\right] \cup\left[e_{1} /(s t), \infty\right]$ and $\left[-e_{1}, e_{1}\right] \mid$. Then $R$ is conformally equivalent to the Teichmüller ring $R_{T, n}\left((1-s t)^{2} /(4 s t)\right)$, and the sphere $S^{n-1}(1 / s)$ separates $R$ into two rings $R_{1}$ and $R_{2}$ that are conformally equivalent to $R_{G, n}\left(\left(1+s^{2}\right) /(2 s)\right)$ and $R_{G, n}\left(\left(1+t^{2}\right) /(2 t)\right)$, respectively. The result now follows from (2.1) and the superadditivity of the modulus [F].

2.8. Corollary. For $n \geq 2, a, b \in(0,1)$,

$$
M_{n}(a)+M_{n}(b) \leq 2 M_{n}(\sqrt{a b}),
$$

where equality holds if and only if $a=b$.

Proof. For $a=b$, equality is clear. Next, for $a \neq b$, let $s=$ $a /\left(1+a^{\prime}\right), t=b /\left(1+b^{\prime}\right)$, where $a^{\prime}=\sqrt{1-a^{2}}, b^{\prime}=\sqrt{1-b^{2}}$. Then 
$a=2 s /\left(1+s^{2}\right), b=2 t /\left(1+t^{2}\right)$, and Theorem 2.7 gives

$$
\begin{aligned}
M_{n}(a)+M_{n}(b) & \leq 2 M_{n}\left(\frac{2 \sqrt{s t}}{1+s t}\right)<2 M_{n}\left(\frac{2 \sqrt{s t}}{\sqrt{\left(1+s^{2}\right)\left(1+t^{2}\right)}}\right) \\
& =2 M_{n}(\sqrt{a b}),
\end{aligned}
$$

since $M_{n}$ is strictly decreasing.

2.9. ReMARK. Corollary 2.8 strengthens [AVV4, (1.22)].

2.10. TheOREM. For each $n \geq 2$, let

$$
f_{n}(r) \equiv \frac{M_{n}(r)}{\log \left(\left(1+r^{\prime}\right) / r\right)} \quad \text { and } \quad g_{n}(r) \equiv \frac{M_{n}(r)}{\log \left(\lambda_{n}\left(1+r^{\prime}\right) /(2 r)\right)} .
$$

Then $f_{n}(r)$ is strictly increasing from $(0,1)$ onto $(1, \infty)$ and $g_{n}(r)$ is decreasing from $(0,1)$ onto $(0,1)$.

Proof. By [LV, (2.5), p. 60] we have

$$
f_{2}(r)=\frac{\mu(r)}{\log \left(\frac{1+r^{\prime}}{r}\right)}=\frac{2 \mu(r)}{\log \left(\frac{1+r^{\prime}}{1-r^{\prime}}\right)}=\frac{\mu(t)}{\log \left(\frac{1}{t}\right)},
$$

where $t=\left(1-r^{\prime}\right) /\left(1+r^{\prime}\right)$, and it follows from [AVV6, Theorem $4.3(4)]$ and $f_{2}(r)$ is increasing from $(0,1)$ onto $(1, \infty)$. For $n \geq 3$ it follows from [AVV4, Lemma 2.6(5)] that the function

$$
f_{n}(r)=f_{2}(r) \cdot \frac{M_{n}(r)}{\mu(r)}
$$

has the desired properties.

Next,

$$
1-g_{n}(r)=\frac{\log \frac{\lambda_{n}}{2}-\left(M_{n}(r)+\log \frac{r}{1+r^{\prime}}\right)}{\log \frac{\lambda_{n}\left(1+r^{\prime}\right)}{2 r}},
$$

which, by Theorem 1.9 , is increasing from $(0,1)$ onto itself.

3. Distortion inequalities in $\mathbb{R}^{n}$. In this section we obtain estimates for the distortion functions $\varphi_{K, n}, \varphi_{K, n}^{*}$, and $\psi_{K, n}$ introduced in (1.4) and (1.5). In [AVV3, Theorem 2.2] it was shown that $\varphi_{K, n}^{*} \neq$ $\varphi_{K, n}$ for $n \geq 3, K \neq 1$. We now show that $\varphi_{K, n}^{*} \neq \psi_{K, n}$ and alse that $\varphi_{K, n}$ and $\psi_{K, n}$ are not comparable. In our first result we apply a method of O. Hübner [H] (cf. [LV, pp. 64, 65], [AVV1, p. 698]) along with our Theorem 1.9 to derive inequalities that improve earlier distortion estimates [AVV1, Theorem 4.10]. 
3.1. Theorem. For $n \geq 2, K \geq 1,0<r<1$, let $\alpha=K^{1 /(1-n)}$, $\beta=1 / \alpha, r^{\prime}=\sqrt{1-r^{2}}, A(r)=r /\left(1+r^{\prime}\right)$. Then

$$
\begin{gathered}
A\left(\varphi_{K, n}(r)\right) \leq\left(\frac{\lambda_{n}}{2}\right)^{1-\alpha} A(r)^{\alpha} \leq K A(r)^{\alpha} \\
A\left(\varphi_{1 / K, n}(r)\right) \geq\left(\frac{\lambda_{n}}{2}\right)^{1-\beta} A(r)^{\beta} \geq K^{-\beta} A(r)^{\beta} \\
\varphi_{K, n}(r) \leq\left(\frac{\lambda_{n}}{2}\right)^{1-\alpha}\left(1+r^{\prime}\right)^{1-\alpha} r^{\alpha} \leq K\left(1+r^{\prime}\right)^{1-\alpha} r^{\alpha} \\
\varphi_{1 / K, n}(r) \geq\left(\frac{\lambda_{n}}{2}\right)^{1-\beta}\left(1+r^{\prime}\right)^{1-\beta} r^{\beta} \geq K^{-\beta}\left(1+r^{\prime}\right)^{1-\beta} r^{\beta}
\end{gathered}
$$

Proof. Let $s=\varphi_{K, n}(r)$. Then $M_{n}(s)=\alpha M_{n}(r), s \geq r$. Hence by Theorem 1.9

$$
\begin{aligned}
\log A(s) & \leq(1-\alpha)\left(M_{n}(r)+\log A(r)\right)+\alpha \log A(r) \\
& \leq(1-\alpha) \log \left(\frac{\lambda_{n}}{2}\right)+\alpha \log A(r) \leq \log K+\alpha \log A(r),
\end{aligned}
$$

and (1) follows. Since $\varphi_{K, n}^{-1}=\varphi_{1 / K, n}$, (2) follows from (1). Finally, (1) implies (3), and (2) implies (4).

3.2. Corollary. For $n \geq 2, K_{2} \geq K_{1}>0, \alpha=\left(K_{2} / K_{1}\right)^{1 /(1-n)}$, $0<r<1$,

$$
\varphi_{K_{2}, n}(r) \leq\left(\frac{\lambda_{n}}{2}\right)^{1-\alpha} \varphi_{K_{1}, n}^{\alpha}(r)\left(1+\sqrt{1-\varphi_{K_{1}, n}^{2}(r)}\right)^{1-\alpha}
$$

Proof. Since $\varphi_{K_{2}, n}(r)=\varphi_{K_{2} / K_{1}, n}\left(\varphi_{K_{1}, n}(r)\right)$, the result follows from $3.1(3)$.

3.3. REMARK. We can obtain analogous inequalities for $\varphi_{K, n}^{*}$ by combining (1.7) with Theorem 3.1.

3.4. Proof of Theorem 1.11. Part (1) follows from Theorem 3.1(1), since $2 \operatorname{arctanh} A(r)=\operatorname{arctanh} r$ and $L A(r)^{\alpha}<1$ if $0<r<r_{1}=$ $\tanh (2 \operatorname{arctanh} l)$. Part (2) follows similarly from Theorem 3.1(2). 
3.5. THEOREM. For $n \geq 2, K \geq 1, \alpha=K^{1 /(1-n)}, \beta=1 / \alpha$, $0<r<1, r^{\prime}=\sqrt{1-r^{2}}$,

(1) $\tanh (\beta \operatorname{arctanh} r) \leq \varphi_{K, n}^{*}(r)$

$$
\begin{aligned}
& \leq \tanh \left(\operatorname{arctanh} r+(\beta-1) M_{n}\left(r^{\prime}\right)\right) \\
& \leq \tanh \left(\beta \operatorname{arctanh} r+(\beta-1) \log \left(\lambda_{n} / 2\right)\right)
\end{aligned}
$$

(2) $\quad \tanh (\alpha \operatorname{arctanh} r) \geq \varphi_{1 / K, n}^{*}(r)$

$$
\begin{aligned}
& \geq \tanh \left(\operatorname{arctanh} r+(\alpha-1) M_{n}\left(r^{\prime}\right)\right) \\
& \geq \tanh \left(\alpha \operatorname{arctanh} r+(\alpha-1) \log \left(\lambda_{n} / 2\right)\right) .
\end{aligned}
$$

The inequalities reduce to equality when $K=1$. Moreover,

$$
\begin{aligned}
& \liminf _{r \rightarrow 0} r^{-\alpha} \operatorname{arctanh} \varphi_{K, n}^{*}(r) \geq 4^{1-\alpha}, \\
& \limsup _{r \rightarrow 0} r^{-\beta} \operatorname{arctanh} \varphi_{1 / K, n}^{*}(r) \leq 4^{1-\beta},
\end{aligned}
$$

$$
\lim _{r \rightarrow 1} \frac{\operatorname{arctanh} \varphi_{K, n}^{*}(r)}{\operatorname{arctanh} r}=\beta, \quad \lim _{r \rightarrow 1} \frac{\operatorname{arctanh} \varphi_{1 / K, n}^{*}(r)}{\operatorname{arctanh} r}=\alpha,
$$

whereas

$$
\begin{aligned}
& \lim _{r \rightarrow 0} r^{-\alpha} \operatorname{arctanh} \varphi_{K, n}(r)=\lambda_{n}^{1-\alpha}, \\
& \lim _{r \rightarrow 0} r^{-\beta} \operatorname{arctanh} \varphi_{1 / K, n}(r)=\lambda_{n}^{1-\beta},
\end{aligned}
$$

(6) $\lim _{r \rightarrow 1} \frac{\operatorname{arctanh} \varphi_{K, n}(r)}{\operatorname{arctanh} r}=K, \quad \lim _{r \rightarrow 1} \frac{\operatorname{arctanh} \varphi_{1 / K, n}(r)}{\operatorname{arctanh} r}=1 / K$.

Proof. From [AVV2, 2.18] and [AVV1, 4.4]

$$
\varphi_{K, n}^{*}(r) \geq \varphi_{\beta, 2}(r) \geq \tanh (\beta \operatorname{arctanh} r)
$$

and

$$
\varphi_{1 / K, n}^{*}(r) \leq \varphi_{\alpha, 2}(r) \leq \tanh (\alpha \operatorname{arctanh} r) .
$$

Next, with $s=\varphi_{K, n}^{*}(r), s^{\prime}=\sqrt{1-s^{2}} \leq r^{\prime}=\sqrt{1-r^{2}}$, Theorem 1.9 and (1.7) give

$$
M_{n}\left(r^{\prime}\right)-\operatorname{arctanh} r \leq M_{n}\left(s^{\prime}\right)-\operatorname{arctanh} s \leq \beta M_{n}\left(r^{\prime}\right)-\operatorname{arctanh} s,
$$

and the second inequality in (1) follows. Then Corollary 1.10(1) yields the third inequality in (1). The proof of the first and second inequalities in (2) is similar.

The first limit in (3) follows from [AVV1, Theorem 4.9] and [LV, p. 65]; and the second follows from the first by inversion. The limits in 
(4) follow directly from (1) and (2); (5) follows from [AVV4, (3.7)]; and (6) is implied by [AVV1, Theorem 4.4].

Theorem 3.5 yields the following sharp quasiconformal distortion result for the hyperbolic metric in $B^{n}$.

3.6. Corollary. If $f \in Q C_{K}\left(B^{n}\right)$ and $\beta=K^{1 /(n-1)}=1 / \alpha$, then

$$
\begin{aligned}
\rho(f(x), f(y)) & \leq \rho(x, y)+2(\beta-1) M_{n}\left(\operatorname{sech} \frac{\rho(x, y)}{2}\right) \\
& \leq \beta \rho(x, y)+2(\beta-1) \log \frac{\lambda_{n}}{2}
\end{aligned}
$$

for all $x, y \in B^{n}$. Moreover, if $f\left(B^{n}\right)=B^{n}$, then we also get the reverse inequality,

$$
\begin{aligned}
\alpha \rho(x & , y)-2(1-\alpha) \log \frac{\lambda_{n}}{2} \\
& \leq \rho(x, y)-2(1-\alpha) M_{n}\left(\operatorname{sech}\left(\frac{\rho(x, y)}{2}\right)\right) \\
& \leq \rho(f(x), f(y)) .
\end{aligned}
$$

Proof. Denoting $\tanh (\rho / 2)=r, \tanh \left(\rho^{\prime} / 2\right)=s, \rho=\rho(x, y)$, $\rho^{\prime}=\rho(f(x), f(y))$, then applying 3.5(1) and [Vu2, 11.2], [MRV], we get

$$
\begin{aligned}
\rho^{\prime} & =2 \operatorname{arctanh} s \leq 2 \operatorname{arctanh} \varphi_{K, n}^{*}(r) \\
& \leq \rho(x, y)+2(\beta-1) M_{n}\left(\operatorname{sech}\left(\frac{\rho(x, y)}{2}\right)\right) \\
& \leq \beta \rho(x, y)+2(\beta-1) \log \frac{\lambda_{n}}{2},
\end{aligned}
$$

proving (1). Inequality (2) follows from (1) applied to $f^{-1}$.

3.7. Corollary. For $K \geq 1, n \geq 2, \beta=K^{1 /(n-1)}=1 / \alpha, 0<$ $r<1$,

$$
\begin{aligned}
& \left(\frac{1+r}{1-r}\right)^{\beta} \leq \frac{1+\varphi_{K, n}^{*}(r)}{1-\varphi_{K, n}^{*}(r)} \leq\left(\frac{\lambda_{n}}{2}\right)^{2(\beta-1)}\left(\frac{1+r}{1-r}\right)^{\beta} \\
& \left(\frac{\lambda_{n}}{2}\right)^{2(\alpha-1)}\left(\frac{1+r}{1-r}\right)^{\alpha} \leq \frac{1+\varphi_{1 / K, n}^{*}(r)}{1-\varphi_{1 / K, n}^{*}(r)} \leq\left(\frac{1+r}{1-r}\right)^{\alpha}
\end{aligned}
$$

Proof. Inequalities (1), (2) follow from Theorem 3.5(1), (2) respectively. 
In a recent paper D. Cooper [C] has shown that, for a class of homeomorphisms of $B^{n}$ onto itself keeping 0 fixed, the image of $S^{n-1}(r)$ is close to $S^{n-1}(r)$ in a certain sense. (For an alternative proof see J. Väisälä [V3].) More precisely, Cooper showed, for a given mapping $f$ in this class and for $r$ close to 1 and $\varepsilon>0$, that $S^{n-1}(r)=A \cup E$, where $A \cap E=\varnothing$ and

$$
f(A) \subset \bigcup_{|z|=r} D(z, \varepsilon) ; \quad D(z, \varepsilon)=\left\{w \in B^{n}: \rho(z, w)<\varepsilon\right\}
$$

and the exceptional set $E$ is small in the $(n-1)$-dimensional measure. Cooper's class of mappings includes quasiconformal mappings in particular. We shall now apply Corollary 3.7 to show that for every $\varepsilon>0$ Cooper's exceptional set $E$ is nonempty for $r$ close to 1 , even for $K$-quasiconformal mappings when $K>1$. Indeed, with $s=\varphi_{K, n}^{*}(r)$ from Corollary 3.7 we obtain

$$
\rho\left(S^{n-1}(r), S^{n-1}(s)\right)=\log \left(\frac{1+s}{1-s} \frac{1-r}{1+r}\right) \geq(\beta-1) \cdot \log \frac{1+r}{1-r},
$$

which tends to $\infty$ as $r \rightarrow 1$. Thus given $K>1$ there exists a $K$-quasiconformal mapping $f$ of $B^{n}$ onto $B^{n}$ keeping 0 fixed and for which there are points on $S^{n-1}(r)$ whose images under $f$ are at hyperbolic distance at least $((\beta-1) / 2) \log ((1+r) /(1-r))$ from $S^{n-1}(r)$. In other words, Cooper's exceptional set is nonempty for all $K>1$ and for all $r$ sufficiently close to 1 .

3.8. Proof of Theorem 1.14. Let $r=\tanh (\rho / 2), s=\tanh \left(\rho^{\prime} / 2\right)$, $r^{\prime}=\sqrt{1-r^{2}}, s^{\prime}=\sqrt{1-s^{2}}$, and $\alpha=K^{1 /(1-n)}$. Then by Theorem 3.1, [AVV4, (3.22)], and the Schwarz lemma [MRV, 3.1],

$$
\begin{aligned}
\tanh \frac{\rho^{\prime}}{4} & =\frac{s}{1+s^{\prime}} \leq \frac{\varphi_{K, n}(r)}{1+\sqrt{1-\varphi_{K, n}(r)^{2}}} \leq\left(\frac{\lambda_{n}}{2}\right)^{1-\alpha}\left(\frac{r}{1+r^{\prime}}\right)^{\alpha} \\
& =\left(\frac{\lambda_{n}}{2}\right)^{1-\alpha}\left(\tanh \left(\frac{\rho}{4}\right)\right)^{\alpha} \leq K\left(\tanh \frac{\rho}{4}\right)^{\alpha} \leq K\left(\tanh \frac{\rho}{4}\right)^{1 / K}
\end{aligned}
$$

When $K=1$ the inequality in the theorem reduces to $\rho^{\prime} \leq \rho$, which by conformal invariance is an equality if $f$ is one-to-one and $f\left(B^{n}\right)=$ $B^{n}$.

3.9. Proof of Corollary 1.15. This follows immediately from Theorem 1.14 and [AVV2, (2.5)]. 
We shall require the following simple inequality (cf. e.g. [Vu2, (2.27)]):

$$
|x-y| \leq 2 \tanh \frac{\rho(x, y)}{4} \leq \frac{|x-y|}{\sqrt{\left(1-|x|^{2}\right)\left(1-|y|^{2}\right)}}
$$

for $x, y \in B^{n}$.

3.11. Proof of Theorem 1.12. For (1), by Theorem 1.14 and (3.10) for $x, y \in \bar{B}^{n}$, with $\rho=\rho(x, y), \rho^{\prime}=\rho(f(x), f(y))$, we have

$$
\begin{aligned}
|f(x)-f(y)| & \leq 2 \tanh \frac{\rho^{\prime}}{4} \leq 2\left(\frac{\lambda_{n}}{2}\right)^{1-\alpha}\left(\tanh \frac{\rho}{4}\right)^{\alpha} \\
& \leq \lambda_{n}^{1-\alpha}\left(1-r^{2}\right)^{-\alpha}|x-y|^{\alpha} .
\end{aligned}
$$

The proof of (2) is similar.

The next result is elementary.

3.12. Lemma. For $K \geq 1$ and $r \in(0,1), r^{\prime}=\sqrt{1-r^{2}}$, the following inequalities hold:

$$
\begin{aligned}
r & <\sqrt{1-r^{\prime 2 K}} \leq \tanh (K \operatorname{arctanh} r) \\
& \leq \min \left\{K r, \sqrt{1-4^{1-K} r^{\prime 2 K}}\right\}
\end{aligned}
$$

$$
\begin{aligned}
\sqrt{\max \left\{\left(\frac{r}{K}\right)^{2}, 1-4^{1-1 / K} r^{2 / K}\right\}} & \leq \tanh \left(\frac{1}{K} \operatorname{arctanh} r\right) \\
& \leq \sqrt{1-r^{\prime 2 / K}}<r
\end{aligned}
$$

3.13. Theorem. For $n \geq 2,0 \leq r \leq 1,1 \leq K<\infty, \beta=$ $K^{1 /(n-1)}, r^{\prime}=\sqrt{1-r^{2}}$,

$$
\sqrt{1-r^{\prime 2 \beta}} \leq \varphi_{K, n}^{*}(r) \leq \psi_{K, n}(r) \leq \sqrt{1-\lambda_{n}^{2(1-\beta)} r^{\prime 2 \beta}} .
$$

Equality holds for $K=1$. The upper bound for $\psi_{K, n}(r)$ is asymptotically sharp as $r$ tends to 1 .

Proof. By Theorem 3.5(1) and Lemma 3.12 we obtain

$$
\varphi_{K, n}^{*}(r) \geq \tanh (\beta \operatorname{arctanh} r) \geq \sqrt{1-r^{\prime 2 \beta}} .
$$

The second inequality follows from (1.7). Next, by (1.4) and Theorem $3.1(4)$ we have

$$
\psi_{K, n}^{2}(r)=1-\varphi_{1 / K, n}^{2}\left(r^{\prime}\right) \leq 1-\lambda_{n}^{2(1-\beta)} r^{\prime 2 \beta},
$$


proving the third inequality. The statement about equality is clear. Finally, the third inequality is asymptotically sharp as $r \rightarrow 1$ since

$$
\lim _{r \rightarrow 1-} r^{\prime-2 \beta}\left(1-\psi_{K, n}^{2}(r)\right)=\lim _{r \rightarrow 1-} r^{\prime-2 \beta} \varphi_{1 / K, n}^{2}\left(r^{\prime}\right)=\lambda_{n}^{2(1-\beta)}
$$

by (1.4) and [AVV1, (4.12)].

3.14. Corollary. Let $n \geq 3, m \geq 2,1<K<\infty$, and $0<r<1$.

(1) If $r$ is close to 1 then $\varphi_{K, n}^{*}(r)<\varphi_{K, m}(r)$.

(2) If $m>n$ and $r$ is close to 0 , then the above inequality is reversed.

Proof. For (1) choose $r$ such that $\left(1-\lambda_{n}^{c}\right)^{1 / 2}<r<1$, where $c=2(1-\beta) /(K-\beta), \beta=K^{1 /(n-1)}$. Then

$$
1-\lambda_{n}^{2(1-\beta)} r^{2 \beta}<1-r^{\prime 2 K}
$$

and the result follows from Lemma 3.12 and Theorem 3.13.

By [AVV1, Theorem 4.10] and [AVV2, (2.18)] we get

$$
\lim _{r \rightarrow 0} \varphi_{K, n}^{*}(r) / \varphi_{K, m}(r)=\infty,
$$

and hence (2) follows.

4. High dimensions. In this section we prove Theorem 1.17, by using the following result from [Vu3].

4.1. Theorem. The linear dilatation in (1.16) has the following majorant:

$$
H_{n}(K) \leq \inf _{0<t<1} \frac{A_{K, n}(t)}{B_{K, n}(t)}
$$

where

$$
A_{K, n}(t)=\frac{\varphi_{K, n}^{2}(\sqrt{t})}{1-\varphi_{K, n}^{2}(\sqrt{t})}, \quad B_{K, n}(t)=\varphi_{1 / K, n}^{2}(\sqrt{t /(1+t)}) .
$$

4.2. Proof of Theorem 1.17. Fix $t \in\left(0,4^{1-K} K^{-2}\right)$. Then by Theorem 3.1(3), (4) and [AVV4, (3.23)],

$$
\begin{aligned}
& \limsup _{n \rightarrow \infty} A_{K, n}(t) \leq \lim _{n \rightarrow \infty} \frac{\lambda_{n}^{2(1-\alpha)} t^{\alpha}}{1-\lambda_{n}^{2(1-\alpha)} t^{\alpha}}=\frac{K^{2} t}{1-K^{2} t}, \\
& \liminf _{n \rightarrow \infty} B_{K, n}(t) \geq \lim _{n \rightarrow \infty} \lambda_{n}^{2(1-\beta)}\left(\frac{t}{1+t}\right)^{\beta}=\frac{t}{K^{2}(1+t)} .
\end{aligned}
$$


Hence

$$
\limsup _{n \rightarrow \infty} \frac{\left.A_{K, n}(t)\right)}{B_{K, n}(t)} \leq \frac{K^{4}(1+t)}{1-K^{2} t}
$$

so that

$$
\limsup _{n \rightarrow \infty} H_{n}(K) \leq \limsup _{n \rightarrow \infty}\left(\inf _{0<t<1} \frac{A_{K, n}(t)}{B_{K, n}(t)}\right) \leq K^{4} .
$$

4.3. Conjectures. (1) $\lim _{n \rightarrow \infty} H_{n}(K)=1$ for all $K>1$.

(2) $\lim _{n \rightarrow \infty} \varphi_{K, n}^{*}(r)=r$ for all $K>1$ and $0<r<1$.

(3) $M_{n}(r)$ can be replaced by $M_{n}^{n-1}(r)$ in Theorem 2.6.

4.4. REMARK. We observe that [AVV1, (4.5), (4.11)] implies that

$$
r<\liminf _{n \rightarrow \infty} \varphi_{K, n}(r) \leq \limsup _{n \rightarrow \infty} \varphi_{K, n}(r) \leq K r
$$

for all $K>1$ and $0<r<1$, so that the analog of 4.3(2) is false for $\varphi_{K, n}(r)$ if $K>1$.

\section{REFERENCES}

[A1] G. D. Anderson, Extremal rings in n-space for fixed and varying $n$, Ann. Acad. Sci. Fenn. Ser. A I, 575 (1974), 1-21.

[A2] - Derivatives of the conformal capacity of extremal rings, Ann. Acad. Sci. Fenn. Ser. A I, 10 (1984), 29-46.

[AF] G. D. Anderson and J. S. Frame, Numerical estimates for a Grötzsch ring constant, Constr. Approx., 4 (1988), 223-242.

[AVV1] G. D. Anderson, M. K. Vamanamurthy, and M. Vuorinen, Dimension-free quasiconformal distortion in $n$-space, Trans. Amer. Math. Soc., 297 (1986), 687-706.

[AVV2] — Sharp distortion theorems for quasiconformal mappings, Trans. Amer. Math. Soc., 305 (1988), 95-111.

[AVV3] _ Inequalities for the extremal distortion function, Proc. 13th Rolf Nevanlinna Colloquium (Joensuu, 1987), Lecture Notes in Math., Vol. 1351, pp. 1-11, Springer-Verlag, Berlin and Heidelberg, New York, 1988.

[AVV4] _ Special functions of quasiconformal theory, Exposition. Math., 7 (1989), 97-138.

[AVV5] _ Distortion functions for plane quasiconformal mappings, Israel $\mathbf{J}$. Math., 62 (1988), 1-16.

[AVV6] __ Functional inequalities for complete elliptic integrals and their ratios, SIAM J. Math. Anal., 21 (1990), 536-549.

[B] A. F. Beardon, The Geometry of Discrete Groups, Graduate Texts in Math. Vol. 91, Springer-Verlag, Berlin, Heidelberg and New York, 1983.

[BF] P. F. Byrd and M. D. Friedman, Handbook of Elliptic Integrals for Engineers and Physicists, Die Grundlehren der math. Wissenschaften, Vol. 57, Springer-Verlag, Berlin, Göttingen and Heidelberg, 1954.

[Ca] E. D. Callendar, Hölder of n-dimensional quasiconformal mappings, Pacific J. Math., 10 (1960), 499-515. 
[C] D. Cooper, Quasi-isometries of hyperbolic space are almost isometries, Manuscript U.C.S.B., September 1988.

[F] B. Fuglede, Extremal length and functional completion, Acta Math., 98 (1957), 171-219.

[G] F. W. Gehring, Symmetrization of rings in space, Trans. Amer. Math. Soc., 101 (1961), 499-519.

[H] O. Hübner, Remarks on a paper by Lawrynowicz on quasiconformal mappings, Bull. de l'Acad. Polon. des Sci., 18 (1970), 183-186.

[LV] O. Lehto and K. I. Virtanen, Quasiconformal Mappings in the Plane, 2nd ed., Die Grundlehren der math. Wissenschaften, Band 126, Springer-Verlag, New York, Heidelberg and Berlin, 1973.

[MRV] O. Martio, S. Rickman and J. Väisälä, Distortion and singularities of quasiregular mappings, Ann. Acad. Sci. Fenn. Ser. AI, 465 (1970), 1-13.

[R] Yu. G. Reshetnyak, Space Mappings With Bounded Distortion, Transl. of Math. Monographs, Vol. 73, Amer. Math. Soc., Providence, RI, 1989.

[T] O. Teichmüller, Untersuchungen über konforme und quasikonforme Abbildung, Deutsche Math., 3 (1938), 621-678.

[V1] J. Väisälä, Lectures on n-Dimensional Quasiconformal Mappings, Lecture Notes in Math. Vol. 229, Springer-Verlag, Berlin, Heidelberg and New York, 1971.

[V2] _ A survey of quasiregular maps in $\mathbb{R}^{n}$, Proc. Internat. Congr. Math. (Helsinki, 1978), Vol. 2, 685-691, Acad. Sci. Fennica, Helsinki, 1980.

[V3] _ Free quasiconformality in Banach spaces II, Ann. Acad. Sci. Fenn. Ser. A. I. Math., 16 (1991), 255-310.

[Vu1] M. Vuorinen, Conformal invariants and quasiregular mappings, J. Analyse Math., 45 (1985), 69-115.

[Vu2] _ Conformal Geometry and Quasiregular Mappings, Lecture Notes in Math., Vol. 1319, Springer-Verlag, Berlin, Heidelberg and New York, 1988.

[Vu3] _ Quadruples and spatial quasiconformal mappings, Math. Z., 205 (1990), 617-628.

Received January 22, 1991 and in revised form January 27, 1992.

Michigan State University

EAST LANSING, MI 48824 U.S.A.

UNIVERSITY OF AUCKLAND

AUCKLAND, New ZeAland

AND

UNIVERSITY OF HELSINKI

Helsinki, Finland 


\section{CONTENTS}

G. D. Anderson, M. K. Vamanamurthy, and M. Vuorinen, Inequalities for quasi-

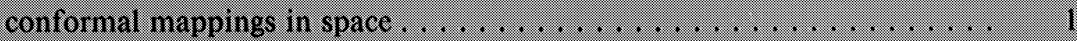

T. Bhattacharya, A nonexistence result for the $n$-Laplacian . . . . . . . . . . . . 19

J. A. Cima, K. Stroethoff, and K. Yale, Bourgain algebras on the unit disk . . . . . 27

J. A. Fridy and C. Orhan, Lacunary statistical convergence . . . . . . . . . . . . . . 43

D. Grenier, On the shape of fundamental domains in $\mathrm{GL}(n, \mathbf{R}) / \mathrm{O}(n) \ldots \ldots . . .53$

B. Jiang and J. Guo, Fixed points of surface diffeomorphisms . . . . . . . . . . . 67

P. Lejarraga, The moduli of rational Weierstrass fibrations over $\mathbf{P}^{\prime}$ : singularities 91

G. J. Martin, On discrete isometry groups of negative curvature . . . . . . . . . . 109

T. Nakashima, Adjoint linear systems on a surface of general type in positive characteristic . . . . . . . . . . . . . . . . . . . . . . . . . 129

B. Ralph, A homotopy transfer for finite group actions $\ldots \ldots \ldots \ldots \ldots \ldots \ldots$

Y. Rong, Maps between Seifert fibered spaces of infinite $\pi_{1} \ldots \ldots \ldots \ldots \ldots, 143$

J.-Y. Shi, Some numeric results on root systems . . . . . . . . . . . . 155

E. Spanier, Singular homology and cohomology with local coefficients and duality

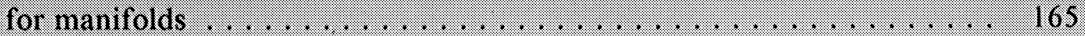




\section{PACIFIC JOURNAL OF MATHEMATICS}

Volume $160 \quad$ No. $1 \quad$ September 1993

Inequalities for quasiconformal mappings in space

GLEN DOUglas ANDERson, MaVina KRISHNa VAMANAMURThy and MATTI VUORINEN

A nonexistence result for the $n$-Laplacian

TILAK BHATTACHARYA

Bourgain algebras on the unit disk

Joseph A. Cima, Karel M. Stroethoff and Keith Yale

Lacunary statistical convergence

JOHN ALBERT FRIDY and CIHAN ORHAN

On the shape of fundamental domains in $\operatorname{GL}(n, \mathbf{R}) / \mathrm{O}(n)$

DOUGLAS MARTIN GRENIER

Fixed points of surface diffeomorphisms

BOJU JIANG and JIANHAN GUO

The moduli of rational Weierstrass fibrations over $\mathbf{P}^{1}$ : singularities

PABLO LEJARRAGA

On discrete isometry groups of negative curvature

GAVEN MARTIN

Adjoint linear systems on a surface of general type in positive characteristic

TOHRU NAKASHIMA

A homotopy transfer for finite group actions

WILLIAM J. RALPH

Maps between Seifert fibered spaces of infinite $\pi_{1}$

YONGWU RONG

Some numeric results on root systems

J. Y. SHI

Singular homology and cohomology with local coefficients and duality for manifolds

EDWIN SPANIER 